\title{
Efeito do consumo de energia na fase pré-puberal sobre o desempenho reprodutivo de marrãs
}

\section{Effect of energy intake in prepuberal age on the reproductive performance of gilts}

\author{
Luis David Solis Murgas' ${ }^{1}$, Ciro Alexandre Alves Torres², Juarez Lopes Donzele ${ }^{1}$
}

\section{Resumo}

Objetivou-se neste estudo avaliar o efeito do consumo de energia na fase pré-puberal sobre o desempenho reprodutivo de 46 marrãs mestiças, com média de idade e peso de 140,7 dias e $63,8 \mathrm{~kg}$, respectivamente. Distribuiram-se as marrãs em quatro tratamentos, com diferentes consumos de energia na fase pré-puberal. Para os consumos de energia, usaram-se quatro tipos de rações contendo os seguintes níveis de energia digestível (ED) por kg: $3.000,3.200,3.400$ e 3.600 kcal. Cada terço das marrãs foi coberta, no primeiro, no segundo e no terceiro cio. Todas as marrãs foram sacrificadas aos 30 dias de gestação para determinação da taxa de ovulação, número de embriões viáveis e mortalidade embrionária. Não houve interação entre o consumo de energia e a ordem de cio em que as marrãs foram cobertas para nenhum dos parâmetros avaliados. A ordem do cio de cobrição afetou a mortalidade embrionária e o número de embriões aos 30 dias de gestação. $O$ consumo de energia teve um efeito quadrático sobre a taxa de ovulação e o número de embriões viáveis. Houve também efeito linear do consumo de energia sobre a mortalidade dos embriões.

Palavras chaves: marrãs; energia; ovulação; embrião

\section{Introdução}

A taxa de ovulação na espécie suína é muito variável, sendo em média de 8 a 16 óvulos. Segundo Warnick et al. (1951), existe em média um aumento de 0,8 óvulos da primeira para a segunda ovulação após a puberdade. A maioria dos trabalhos realizados com marrãs destinadas à reposição do plantel, visa a promover maior taxa de ovulação e conseqüentemente aumento do número de leitões nascidos, mediante o fornecimento de alimentação rica em energia por períodos bastante variáveis (Gomes et al. 1982). A adição de energia às rações das marrãs, por períodos de aproximadamente oito a dez dias, mas não menos do que quatro dias antes do cio, tem sido reportada como causadora de um aumento na taxa de ovulação em marrãs (Anderson e Melampy 1972, Brooks e Cooper 1972, Dailey et al. 1975). Goode et al. (1965) verificaram que a taxa de ovulação foi menor nos animais que consumiram uma ração de elevada energia digestiva (ED) $(9.190 \mathrm{kcal}$ de ED/dia). Esses resultados diferem dos obtidos por Haines et al. (1959), Zimmerman et al. (1960a) e Sorensen et al. (1961).

A perda embrionária é um fator importante para o aumento do tamanho da leitegada. Estimativas das perdas embrionárias compiladas por diversos autores indicam que $40 \%$ dos ovos são perdidos antes do parto. Um alto nível de energia durante o período embrionário tem levado a um aumento na mortalidade embrionária. Robertson et al. (1951), Self et al. (1955), Gossett e Sorensen (1959) e Frobish (1970) constataram que a ingestão de energia durante o início da gestação pode ser reduzida a níveis abaixo do normal sem causar efeito sobre a reprodução. Além da taxa de ovulação, a capacidade uterina é outro fator importante que afeta a mortalidade embrionária. Altas taxas de ovulação têm sido associadas com uma grande mortalidade embrionária (Anderson e Melampy 1972).

A relação existente entre a taxa de ovulação, mortalidade embrionária e performance reprodutiva, particularmente tamanho da leitegada em marrãs, não tem sido estudada nas condições de alimentação dos animais para reposição do plantel.

\section{Material e Métodos}

O experimento foi conduzido na Seção de Suínocultura do Departamento de Zootecnia do Centro de Ciências Agrárias da Universidade Federal de Viçosa, localizada no município de Viçosa, Minas Gerais. Utilizaram-se 46 marrãs mestiças com idade e peso inicial médios de 140,7 dias e 63,8 kg, agrupadas de acordo com o peso e grau de parentesco. As marrãs foram alojadas em grupo de 8 , em baias de alvenaria com solário de $12 \mathrm{~m}^{2}$ de área.

\footnotetext{
Trabalho extraído da Tese de Mestre em Reprodução Animal do primeiro autor, apresentada ao Departamento de Zootecnia da Universidade Federal de Viçosa, UFV.

1 Departamento de Medicina Veterinária, Universidade Federal de Lavras, UFLA, MG, Brasil

${ }^{2}$ Departamento de Zootecnia, UFLA
} 
O delineamento experimental usado foi o inteiramente casualizado, com quatro tratamentos, sendo a unidade experimental constituída de um animal. Os tratamentos foram constituídos a partir das quantidades de energia consumida pelas marrãs durante a fase pré-púbere, e para obtenção deles foram fornecidos às marrãs quatro tipos de rações contendo diferentes níveis de energia: 3.000 , $3.200,3.400$ e $3.600 \mathrm{kcal} / \mathrm{ED} / \mathrm{kg}$, respectivamente.

As rações experimentais (Tabela1) foram formuladas a base de milho e farelo de soja, suplementadas com minerais e vitaminas de modo a atender às exigências nutricionais de suínos em terminação, segundo Rostagno et al. (1983), exetuando-se o nível de ED, que foi ajustado com areia lavada e/ou, óleo de soja em substituição ao amido da ração.

Tabela 1 - Composição das rações experimentais

\begin{tabular}{lrrrr}
\hline \multirow{2}{*}{ Ingrediemtes } & \multicolumn{4}{c}{ Rações } \\
\cline { 2 - 5 } & T-1 & T-2 & T-3 & T-4 \\
\hline Milho & 65,70 & 65,70 & 65,70 & 65,70 \\
Farelo de soja & 20,90 & 20,90 & 20,90 & 20,90 \\
Amido & - & 5,40 & 5,73 & 1,05 \\
Óleo de soja & - & - & 2,37 & 7,05 \\
Arreia lavada & 10,80 & 5,40 & 2,70 & 2,70 \\
Fosfato bicálcico & 1,30 & 1,30 & 1,30 & 1,30 \\
Calcário & 0,80 & 0,80 & 0,80 & 0,80 \\
Sal comum & 0,35 & 0,35 & 0,35 & 0,35 \\
Sup. vitamínico (1) & 0,10 & 0,10 & 0,10 & 0,10 \\
Sup. mineral (2) & 0,05 & 0,05 & 0,05 & 0,05 \\
\hline Total & 100,00 & 100,00 & 100,00 & 100,00 \\
\hline Composição calculada $(3)$ & & & \\
\hline Proteína bruta (\%) & 15,10 & 15,10 & 15,10 & 15,10 \\
Energia digestível & & & & \\
(kcal/kg) & 3.000 & 3.200 & 3.400 & 3.600 \\
Lisina (\%) & 0,750 & 0,750 & 0,750 & 0,750 \\
Cálcio (\%) & 0,677 & 0,677 & 0,677 & 0,677 \\
Fósforo total (\%) & 0,513 & 0,513 & 0,513 & 0,513 \\
\hline
\end{tabular}

(1) - Rovimix Roche: contém por kg do produto: vitamina $A$ $12.000 .000 \mathrm{Ul}$; vitamina $\mathrm{D}_{3} 1.500 .000 \mathrm{Ul}$; vitamina $\mathrm{E} 8.000 \mathrm{Ul}$; vitamina $K 34 \mathrm{~g}$; vitamina $B 2 \mathrm{~g}$; vitamina $B_{2} 4 \mathrm{~g}$; vitamina $B_{6} 5 \mathrm{~g}$; vitamina B12 $30.000 \mathrm{mcg}$; ácido nicotínico $40 \mathrm{~g}$; ácido pantotênico $20 \mathrm{~g}$; bacitracina de zinco $10 \mathrm{~g}$; antioxidante $30 \mathrm{~g}$; selenito de sódio $50 \mathrm{mg}$; excipiente q.s.p. $1.000 \mathrm{~g}$.

(2) - Roligomix Suínos, Roche: contém por kg do produto: ferro $180 \mathrm{~g}$; cobre $20 \mathrm{~g}$; cobalto $4 \mathrm{~g}$; manganês $80 \mathrm{~g}$; zinco $140 \mathrm{~g}$; iodo $4 \mathrm{~g}$; excipiente q.s.p. $1.000 \mathrm{~g}$.

(3) Composição calculada segundo Rostagno et al. (1983).

No momento do primeiro cio, um terço das marrãs de cada tratatamento foram separadas e cobertas com varrões de fertilidade conhecida. Um terço das marrãs foram cobertas no segundo cio e outro terço no terceiro cio; todas as marrãs do experimento foram sacrificadas com 30 dias de gestação para se verificar a taxa de ovulação por meio da contagem dos corpos lúteos presentes nos ovários, o número, peso e comprimento dos embriões presentes no útero, assim como o peso do útero.

Os dados coletados foram submetidos à análise de variância, utilizando o método dos quadrados mínimos no sistema SAEG (Sistema de Análises Estatísticas e Genéticas) desenvolvido pela Universidade Federal de Viçosa. O modelo estatístico utilizado foi o seguinte:

onde

$$
Y_{i j k}=m+T_{i}+C_{j}+(E X C)_{i j}+E_{i j k}
$$

$\mathrm{Y}_{\mathrm{ijk}}=$ observação feita na marrã $\mathrm{k}$ do tratamento $\mathrm{i}$, na ordem do cio de cobrição j

$i=1,2,3,4$

$\mathrm{j}=$ primeiro, segundo e terceiro cios

$\mathrm{m}=$ média

$T_{i}=$ efeito do tratamento $i$ sendo $i=1,2,3,4$

$\mathrm{C}_{\mathrm{j}}=$ efeito do cio de cobrição $\mathrm{j}$ (primeiro, segundo $\mathrm{e}$ terceiro cios)

$(E X C)_{i j k}=$ interação do consumo de energia (tratamentos) sobre o cio de cobrição das marrãs

$E_{i j k}=$ erro associado a cada observação.

\section{Resultados e Discussão}

Os resultados do desempenho reprodutivo obtidos de marrãs abatidas aos 30 dias de gestação, de acordo com o consumo médio diário de energia na fase pré-púbere estão apresentados na Tabela 2. Não foi observada interação $(P>0,10)$ entre o consumo de energia e a ordem do cio em que as marrãs foram cobertas para nenhum dos parâmetros avaliados.

Observou-se efeito quadrático do consumo de energia sobre a taxa de ovulação que aumentou $(P<0,03)$ até o consumo médio diário de energia de $9.775 \mathrm{kcal} / \mathrm{ED} / \mathrm{dia}$ (Fig.1), e sobre o número de embriões viáveis aos 30 dias de gestação que aumentou $(P<0,06)$ até o consumo médio diário de $9.576 \mathrm{kcal} / \mathrm{ED} / \mathrm{dia}$ (Fig. 2). Ocorreu também efeito $(P<0,06)$ do consumo médio diário de energia sobre a mortalidade embrionária que aumentou linearmente (Fig. 3).

Não foram verificados efeitos $(P>0,10)$ do consumo médio diário de energia sobre o peso e comprimento dos embriões e nem sobre o peso do útero das marrãs aos 30 dias de gestação. Os resultados aqui apresentados foram semelhantes aos de Robertson et al. (1951), Zimmerman et al. (1960b), Dailey et al. (1975), Anderson e Melampy (1972) e Cox et al. (1987). Postula-se que um maior consumo de energia possa exercer um efeito positivo sobre a liberação dos hormônios que estão relacionados com o processo ovulatório na espécie suina mas que também existe um limite para esse consumo de energia que, quando ultrapassado provoca um efeito negativo sobre a taxa de ovulação. A redução do número de embriões aos 30 dias de gestação, observada nas marrãs que consumi- 
Tabela 2- Taxa de ovulação, mortalidade embrionária, número, peso e comprimento dos embriões e peso do útero das marrãs aos 30 dias de gestação, consumindo diferentes níveis de energia na fase pré-púbere

Parâmetros

Consumo médio de energia (kcal/ED/dia)

\begin{tabular}{|c|c|c|c|c|c|}
\hline & $\begin{array}{c}\mathrm{T} 1 \\
(8.840)\end{array}$ & $\begin{array}{c}\mathrm{T} 2 \\
(9.430)\end{array}$ & $\begin{array}{c}\text { T3 } \\
(10.020)\end{array}$ & $\begin{array}{c}\mathrm{T} 4 \\
(10.610)\end{array}$ & $\mathrm{CV}(\%)$ \\
\hline Número de marrãs & 12 & 12 & 11 & 11 & - \\
\hline $\begin{array}{l}\text { Taxa de ovulação }(\mathrm{CL})^{1} \\
\text { Mortalidade embrionária }(\%)^{2} \\
\text { Número de embriões viáveis }{ }^{3} \\
\text { Peso dos embriões }(\mathrm{g}) \\
\text { Comprimento dos embriões }(\mathrm{g}) \\
\text { Peso do útero }(\mathrm{g})\end{array}$ & $\begin{array}{r}13,3 \pm 0,68 \\
8,8 \pm 3,58 \\
12,1 \pm 0,62 \\
6,7 \pm 0,28 \\
2,6 \pm 0,36 \\
1.786,3 \pm 0,98\end{array}$ & $\begin{array}{r}14,8 \pm 0,68 \\
16,5 \pm 3,58 \\
12,4 \pm 0,62 \\
6,2 \pm 0,28 \\
2,6 \pm 0,36 \\
1.816,2 \pm 0,98\end{array}$ & $\begin{array}{r}15,1 \pm 0,68 \\
13,7 \pm 3,67 \\
13,1 \pm 0,62 \\
6,8 \pm 6,28 \\
2,7 \pm 0,26 \\
1.902,2 \pm 0,98\end{array}$ & $\begin{array}{r}13,7 \pm 0,68 \\
20,7 \pm 3,67 \\
11,0 \pm 0,68 \\
6,0 \pm 0,32 \\
2,6 \pm 0,40 \\
1.788,3 \pm 0,98\end{array}$ & $\begin{array}{r}19,6 \\
82,7 \\
17,5 \\
15,2 \\
4,8 \\
18,6\end{array}$ \\
\hline
\end{tabular}

1 e 3: efeito quadrático $(P<0,03)$ e $(P<0,06)$, respectivamente; 2: efeito linear $(P<0,06)$; $C V$ : coeficiente de variância.

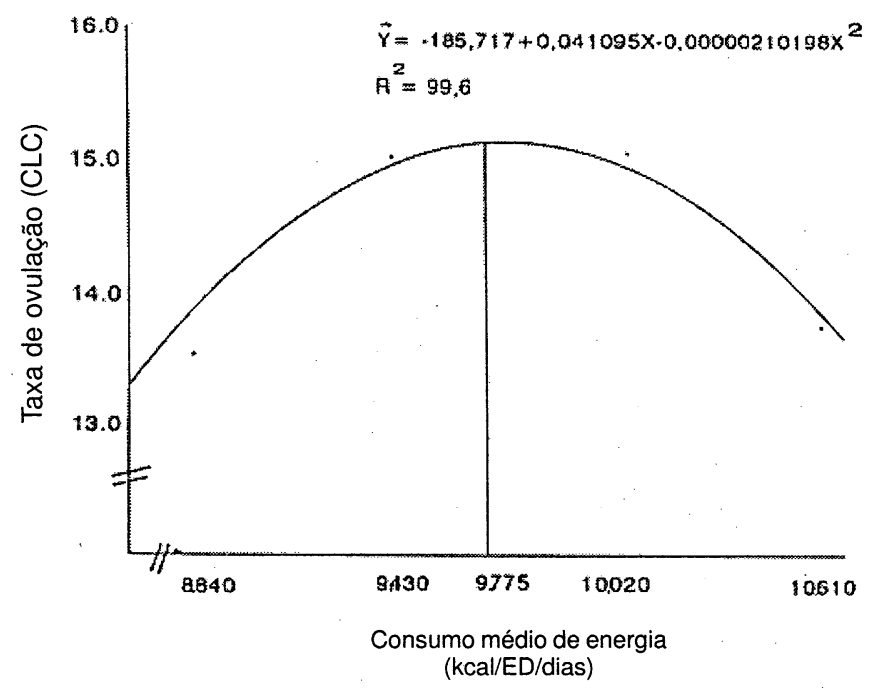

Figura 1 - Taxa de ovulação observada aos 30 dias de gestação em marrãs consumindo diferentes níveis de energia durante a fase prépúbere.

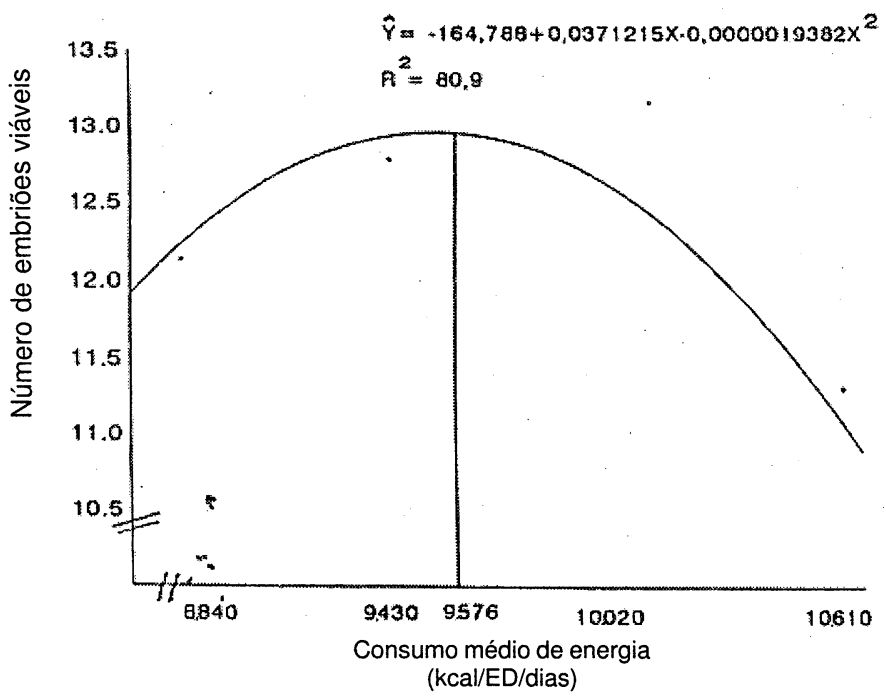

Figura 2 - Número de embriões viáveis encontrados aos 30 dias de gestação em marrãs que consumiram diferentes níveis de energia durante a fes pré-púbere.

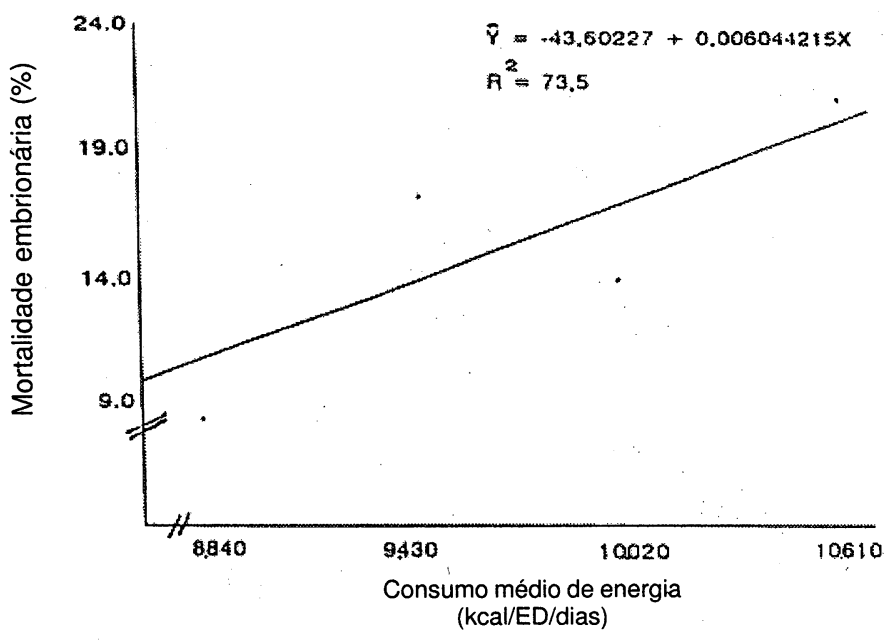

Figura 3 - Mortalidade embrionária observada aos 30 dias de gestação em marrãs consumindo diferentes níveis de energia durante a fase pré-púbere.

ram os níveis mais altos de energia, sugere que isto seja decorrente do efeito sobre a taxa de ovulação que pode estar relacionado a um menor peso uterino observado nos animais que tiveram maior consumo de energia, ainda que essas diferenças não fossem significativas. Os resultados obtidos para o peso e comprimento dos embriões revelam que o desenvolvimento dos embriões até os 30 dias de gestação ocorre independentemente do consumo de energia na fase pré-púbere e nos níveis estudados.

Os resultados da taxa de ovulação, mortalidade embrionária, número, peso e comprimento dos embriões e peso do útero das marrãs aos 30 dias de gestação, de acordo com a ordem do cio de cobrição dos animais, encontram-se na Tabela 3.

Não ocorreu efeito $(P>0,10)$ da ordem do cio de cobrição das marrãs sobre a taxa de ovulação, peso e comprimento dos embriões. Entretanto, a ordem do cio de cobrição afetou $(P<0,10)$ a mortalidade embrionária, o número de embriões viáveis e o peso do útero das 
Tabela 3- Taxa de ovulação, mortalidade embrionária, número, peso e comprimento dos embriões e peso do útero das marrãs aos 30 dias de gestação, e idade e pesos à cobrição no primeiro, segundo e terceiro cios

\begin{tabular}{lccr}
\hline Parâmetros & \multicolumn{3}{c}{ Ordem do cio de cobrição } \\
\cline { 2 - 4 } & Primeiro & Segundo & Terceiro \\
\hline Número de marrãs & 16 & 15 & 15 \\
Taxa de ovulação & $13,4 \pm 0,56 \mathrm{a}$ & $14,3 \pm 0,56 \mathrm{a}$ & $15,0 \pm 0,56 \mathrm{a}$ \\
Mortalidade embrionária (\%) & $18,8 \pm 3,10 \mathrm{a}$ & $16,8 \pm 3,10 \mathrm{ab}$ & $9,1 \pm 3,23 \mathrm{~b}$ \\
Número de embriões viáveis & $10,8 \pm 0,53 \mathrm{a}$ & $11,9 \pm 0,53 \mathrm{ab}$ & $13,8 \pm 0,56 \mathrm{~b}$ \\
Peso dos embriões $(\mathrm{g})$ & $6,7 \pm 0,25 \mathrm{a}$ & $6,1 \pm 0,26 \mathrm{a}$ & $6,6 \pm 0,26 \mathrm{a}$ \\
Comprimento dos embriões (g) & $2,6 \pm 0,32 \mathrm{a}$ & $2,6 \pm 0,33 \mathrm{a}$ & $2,6 \pm 0,33 \mathrm{a}$ \\
Peso do útero (g) & $1.640,0 \pm 0,85 \mathrm{a}$ & $1.778,0 \pm 0,85 \mathrm{a}$ & $2.122,0 \pm 0,88 \mathrm{~b}$ \\
Idade à cobrição (dias) & $200,3 \pm 3,6 \mathrm{a}$ & $225,2 \pm 3,5 \mathrm{~b}$ & $245,7 \pm 3,6 \mathrm{c}$ \\
Peso à cobrição $(\mathrm{kg})$ & $105,4 \pm 2,5 \mathrm{a}$ & $119,4 \pm 2,4 \mathrm{~b}$ & $129,8 \pm 2,4 \mathrm{c}$ \\
\hline
\end{tabular}

Média seguida das mesmas letras na mesma linha não diferem, significativamente, a $5 \%$ de probabilidade pelo teste Tukey.

marrãs $(P<0,01)$ aos 30 dias de gestação. Embora não tenha sido observado efeito da ordem do cio de cobrição sobre a taxa de ovulação, as marrãs cobertas no segundo cio tiveram, em média, 0,9 óvulos a mais do que as cobertas no primeiro cio, enquanto que as cobertas no terceiro cio tiveram, em média, 0,7 óvulos a mais do que as cobertas no segundo cio. Resultados similares foram obtidos por Zimmerman et al. (1960b) e Kirkwood eAherne (1985). Estes últimos concluíram que a taxa de ovulação nas marrãs que atingem a puberdade em idades precoces poderá aumentar a cada novo período de cio após a puberdade e nas marrãs que a retardam não é observado aumento significativo da taxa de ovulação nos cios subseqüentes à puberdade. Apesar da tendência de menor taxa de ovulação, as marrãs cobertas no primeiro cio apresentaram maior $(P<0,10)$ porcentagem de mortalidade embrionária que pode ser o reflexo de um inadequado desenvolvimento uterino.

Além de afetar a mortalidade embrionária, a ordem do cio de cobrição influênciou $(P<0,01)$ o número de embriões viáveis aos 30 dias de gestação, sendo que as marrãs cobertas no terceiro cio tiveram maior número de embriões em relação àquelas cobertas no primeiro cio, e as cobertas no segundo cio tiveram um número de embriões intermediário que não diferiu dos outros dois grupos. Os resultados do presente estudo revelam que o aumento significativo do número de embriões viáveis aos 30 dias de gestação, do primeiro para o terceiro cio, está proporcionalmente mais relacionado a uma queda da mortalidade embrionária do que ao aumento da taxa de ovulação, uma vez que esta não variou significativamente com a ordem do cio de cobrição das marrãs.

Foi observada uma relação direta entre o peso do útero das marrãs aos 30 dias de gestação e o número de embriões viáveis, sugerindo que o tamanho do útero pode desenvolver um papel importante sobre o número de embriões e, conseqüentemente, o número de leitões nascidos.

\section{Conclusões}

Os resultados obtidos permitem concluir que: (1) o aumento no consumo de energia em marrãs, durante a fase pré-pubere, aumenta a mortalidade embrionária e influencia a taxa de ovulação e o número de embriões viáveis; (2) as marrãs cobertas no terceiro cio apresentaram maior peso do útero em relação àquelas cobertas no primeiro cio, apresentando também menor percentagem de mortalidade embrionária e maior número de embriões viáveis, o que mostra o efeito da capacidade uterina sobre o desempenho reprodutivo.

\section{Abstract \\ Effect of energy intake in prepuberal age on the re- productive performance of gilts}

This experiment was conducted to evaluate the effect of energy intake on the reproductive performance of 46 crossbred Large-White $\times$ Landrace gilts, averaging 140.7 days of age and $63.8 \mathrm{~kg}$ of body weight. The gilts were ramdonly distributed into four treatments based on different amount of energy intake at pubertal phase. Four different types of rations were used with energy levels of $3,000,3,200,3,400$, and $3,600 \mathrm{kcal}$ of digestible energy per $\mathrm{kg}$. Each one third of the gilts were bred at the first, second and third estrus. All gilts were slaughtered at the thirty days postbreeding, in order to evaluate the ovulation rate, numbers of viable embryos and embryo mortality rate. None of the parameters studied were affected by the interaction between energy intake and the order of the breeding estrus of the gilts. The breeding estrus order affected the embryo mortality rate and the number of embryos on the thirty day postbreeding. The energy intake affected quadratically the ovulation rate and the number of viable embryos. A linear effect of the energy intake ovær embryo mortality rate was detected.

Key words: gilts; energy; ovulation; embryo 


\section{Referências bibliográficas}

Anderson LL, Melampy RM 1972. Factors affecting ovulation rate in the pig, p. 329-366. In DJA Cole Pig Production, Butterworth, London.

Brooks PH, Cole DJA 1972. The effect on litter size of increased feed intake during the oestrus period in the sow. Animal Production 14(2): 241-245.

Cox NM, Stuart MJ, Althen TG, Bennett WA, Iller HW 1987. Enhancement of ovulation rate in gilts by increasing dietary energy and administering insulin during follicular growth. $J$ An Sci 64(2): 507-509.

Dailey RA, Clark JR, First NL, Chapman AB, Casida LE 1975. Effect of short-term flushing on follicular development at estrus and ovulation rate of gilts of different genetic groups. J An Sci 41(3): 842-847.

Frobish LT 1970. Effect of energy intake on reproductive performance and estrous synchronization of gilts. J An Sci 31(3): 486-490.

Gomes PC, Ferreira AS, Fialho ET, Costa V 1982. Influência da quantidade de ração fornecida a marrãs, do primeiro ao terceiro cio, sobre o desempenho reprodutivo. Rev Soc Bras Zoo 11(2): 252-261.

Goode L, Warnick AC, Wallace HD 1965. Effect of dietary energy level upon reproduction and the relation of endometrial phosphatase activity to embryo survival in gilts. J An Sci 24(4): 959-963.

Gossett JW, Sorensen AM 1959. The effects of two levels of energy and seasons on reproductive phenomena of gilts. J An Sci 18(1): 40-47.
Haines CE, Warnick AC, Wallace, HD 1959. The effect of two levels of energy intake on reproductive phenomena in Duroc Jersey gilts. J An Sci 18(1): 347-354.

Kirkwood RN, Aherne FX 1985. Energy intake, body composition and reproductive performance of the gilt. $J$ An Sci 60(6): 518-1529.

Robertson GL, Grummer RH, Casida LE, Chapman AB 1951. Age at puberty and related phenomena in outbreed Chester White and Poland China gilts. JAn Sci 10(3): 647-656.

Rostagno HS, Silva DJ, Costa PMA, Fonseca JB, Pereira JAA, Silva MA 1983. Composição de Alimentos e Exigências Nutricionais de Aves e Suinos (Tabelas brasileiras), Universidade Federal de Viçosa, Viçosa, 60 pp.

Self HL, Grummer RH, Casida LE 1955. The effects of various sequences of full and limited feeding on the reproductive phenomena in Chester White and Poland China gilts. $J$ An Sci 14(2): 573-592.

Sorensen AM, Thomas WB, Gosset JW 1961. A further study of level of energy intake and season on reproductive performance of gilt. J An Sci 20(2): 347-349.

Warnick AC, Wiggins EL, Casida LE, Grummer RH, Chapman $A B$ 1951. Variation in puberty phenomena in inbred gilts. J An Sci 10(3): 479-493.

Zimmerman DR, Spies HG, Rigor EM, Self HL, Casida LE 1960. Effect of restricted feeding, crossbreeding and season of birth on age at puberty in swine. J An Sci 19(3): 687-694.

Zimmerman DR, Spies HG, Self HL, Casida LE 1960a. Ovulation rate in swine as afected by increased energy intake just prior to ovulation. J An Sci 19(1): 295-301. 